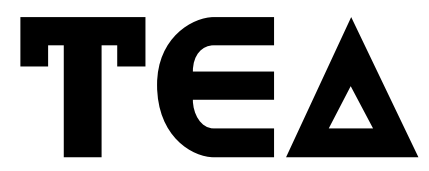

TECNE, EPISTEME Y DIDAXIS

\title{
Revista de la Facultad de Ciencia y Tecnología
}

Investigación y experiencias didácticas de las ciencias experimentales, de las matemáticas y de las tecnologías.

Número 28, Segundo semestre de 2010 ISSN 0121-3814

Clasificada en COLCIENCIAS en categoría B

\author{
Juan Carlos Orozco Cruz \\ Rector \\ Edgar Alberto Mendoza Parada \\ Vicerrector Académico \\ José Domingo Garzón \\ Vicerrector de Gestión Universitaria \\ María Ruth Martínez Hernández \\ Vicerrectora Administrativa y financiera \\ Luis Eduardo Espitia Supelano \\ Decano de la Facultad de Ciencia y Tecnología \\ Director / Editor \\ Diana Lineth Parga Lozano \\ Profesora del Departamento de Química \\ Subdirectora / Coeditora \\ María Nubia Soler Álvarez \\ Profesora del Departamento de Matemáticas
}
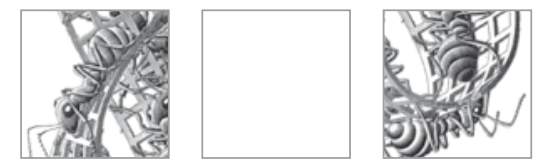

\section{Universidad Pedagógica Nacional}

Facultad de Ciencia y Tecnología Calle 72 No. 11-86 Oficina B-223

Fax 5941894 Ext. 413 www.pedagogica.edu.co

resvistated@pedagogica.edu.co revistated.fct @gmail.com

Revista dirigida a: Investigadores, profesores y estudiantes de didáctica de las ciencias experimentales, de las matemáticas y de las tecnologías.
Indexada en:

Educational Research Abstract, ERA Índice de Revistas de Educación Superior e Investigación Educativa, IRESIE

Catálogo Latindex-Sistema Regional de Información en Línea para Revistas Científicas de América Latina, el Caribe, España y Portugal

Chemical Abstracts, Índice internacional de Citas Latinoamericanas en Ciencias Sociales y Humanidades (Clase) de la Universidad Autónoma de México

Biological Abstracts

Biblioteca digital OEl http://www. campus-oei.org/oeivirt/ 


\section{Comité editorial}

Magíster Rómulo Gallego Badillo

Profesor Departamento de Química

Magíster Royman Pérez Miranda

Profesor Departamento de Química

Doctora Carmen Alicia Martínez Rivera Universidad Distrital Francisco José de Caldas

Doctora Adriana Patricia Gallego

Universidad Distrital Francisco José de caldas

Doctora Rosalba Pulido de Castellanos

Profesora Departamento de Biología

Magíster Lola Constanza Melo Salcedo

Profesora Departamento de Biología

\section{Magíster Isabel Garzón}

Profesora Departamento de Física

Luis Eduardo Espitia Supelano

Decano Facultad de Ciencia y Tecnología

Doctor Alfonso Claret Zambrano

Universidad del Valle (Colombia)

Doctor Leonardo Fabio Martínez

Profesor Departamento de Química

Doctor Luis Bayardo Sanabria

Profesor Departamento de Tecnología

Magíster Edgar Guacaneme

Profesor Departamento de Matemáticas

Magíster Juan Carlos Orozco Cruz

Profesor Departamento de Física

\section{Magíster Néstor Méndez}

Profesor Departamento de Física
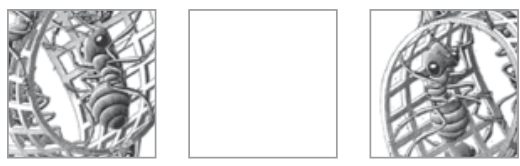

Corrección de estilo

Juliana Avella

Diseño y Diagramación

Víctor Leonel Gómez R.

Elaboración y revisión de abstract

Magíster Deisy Baracaldo

Lingüista aplicada a la enseñanza del inglés

Apoyo editorial

Darwin Leonardo Vargas

\section{Comité asesor}

Doctor Agustín Adúriz-Bravo

Universidad de Buenos Aires (Argentina)

Doctor Andoni Garritz Ruiz

Universidad Nacional Autónoma de México

Doctor Eduardo González

Universidad Nacional de Córdoba (Argentina)

Doctor Mario Quintanilla Gatica

Pontificia Universidad Católica de Chile

Doctor Pedro da Cunha Pinto Neto

Universidad Estatal de Campinas (Brasil)

Doctor Pedro Membiela

Universidad de Vigo (España)

Doctor Roberto Figueroa Molina

Universidad del Atlántico (Colombia)

Doctora Anna María Pessoa de Carvalho

Universidad de Sao Paulo (Brasil)

Doctora Fanny Angulo Delgado

Universidad de Antioquia (Colombia)

Doctora Graciela Utges

Universidad Nacional de Rosario (Argentina)

Doctora Lilia Reyes Herrera

Profesora Doctorado en Educación

Magíster Dino Segura R

Escuela Pedagógica Experimental (Colombia)

Magíster Hernán Díaz Rojas

Profesor Departamento de Matemáticas

Magíster María Laura Eder

Universidad de Buenos Aires (Argentina)

Doctora Julia Salinas

Universidad de Tucumán (Argentina)

Doctor Bruno D’ Amore

Universitá di Bologna (Italia)

Doctor Antonio Cachapuz

Universidad de Aveiro (Portugal)

Magíster Gloria García de García

Profesora Departamento de Matemáticas

Doctor Jaime Sánchez

Universidad de Chile

Doctor Fidel Antonio Cárdenas Salgado

Universidad Pedagógica Nacional

Doctor Roberto Nardi

Universidad Estatal Paulista, Campus de Bauru (Brasil)

Doctor Daniel Gil Pérez

Universidad de Valencia (España)

Doctor João Batista Siqueira Harres

Centro Universitario UNIVATES (Brasil)

Doctor Fernando Bastos

Universidad Estatal Paulista, Campus de Bauru (Brasil)

Doctor Rafael Porlán Ariza

Universidad de Sevilla (España)

Doctora Amparo Vilches

Universidad de Valencia (España) 


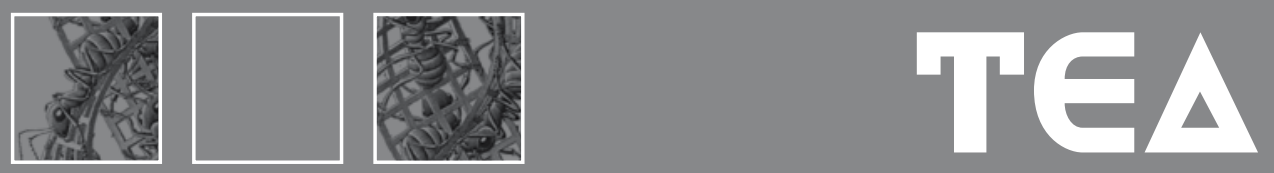

\section{Contenido}

\section{Editorialn \\ Artículos:}

Una aplicación de redes sistémicas para entender las concepciones de los estudiantes: ¿qué tan grande es un átomo? Diana M. Farías | Manuel F. | Molina José G. Carriazo.

20 Descripción de textos escolares en tomo al concepto de función a trozos Carolina Luque.

Concepciones sobre ciencia e investigación en profesores de 41 química en formación inicial: un estudio en el contexto de los trabajos experimentales

Olga Lucia Borda Prada | Manuel Erazo Parga.

Dificultades de los profesores para integrar el uso de Cabri en clase 57 de geometría. Experiencias de un curso de formación docente Martin Eduardo Acosta Gempeler.

73

88

Conociendo el mundo de los polímeros: unidad didáctica como

Algunas relaciones entre algoritmos y resolución de problemas Jaime Fonseca González | Brigitte Johana Sánchez Robayo.

una estrategia para el aprendizaje $\mathbf{\square}$

Martha Janneth Saavedra Alemán | Yolanda Ladino Ospina | Leidy Moreno Rodriguez

101

Matematización de los procesos químicos. Segunda parte.

Rómulo Gallego Badillo| Royman Pérez Miranda | Adriana Patricia Gallego Torres

110 Tesis de Maestría 